\title{
Acceleration and Velocity Sensing from Measured Strain
}

\author{
Chan-gi Pak ${ }^{1}$ and Roger Truax ${ }^{2}$ \\ NASA Armstrong Flight Research Center, Edwards, CA 93523-0273
}

\begin{abstract}
A simple approach for computing acceleration and velocity of a structure from the strain is proposed in this study. First, deflection and slope of the structure are computed from the strain using a two-step theory. Frequencies of the structure are computed from the time histories of strain using a parameter estimation technique together with an autoregressive moving average model. From deflection, slope, and frequencies of the structure, acceleration and velocity of the structure can be obtained using the proposed approach. Simple harmonic motion is assumed for the acceleration computations, and the central difference equation with a linear autoregressive model is used for the computations of velocity. A cantilevered rectangular wing model is used to validate the simple approach. Quality of the computed deflection, acceleration, and velocity values are independent of the number of fibers. The central difference equation with a linear autoregressive model proposed in this study follows the target response with reasonable accuracy. Therefore, the handicap of the backward difference equation, phase shift, is successfully overcome.
\end{abstract}

\section{Nomenclature}

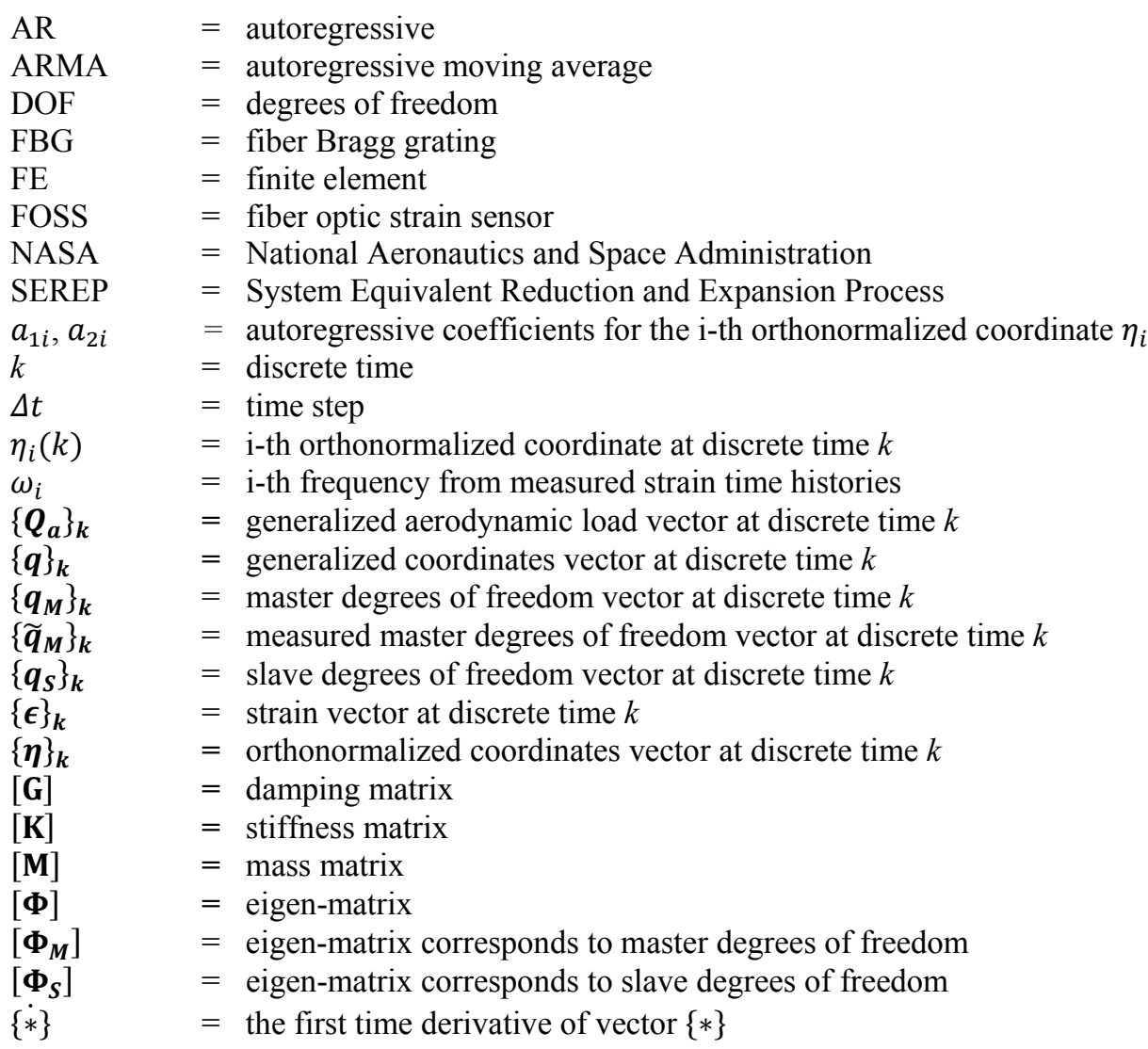

\footnotetext{
${ }^{1}$ Senior Aerospace Engineer, Aerostructures Branch, P.O. Box 273 Edwards, California/Mailstop 48201A, Senior Member AIAA.

${ }^{2}$ Aerospace Engineer, Aerostructures Branch, P.O. Box 273 Edwards, California/Mailstop 48201A, non-member.
} 
$\begin{array}{ll}\{\ddot{*}\} & =\text { the second time derivative of vector }\{*\} \\ {[*]^{-1}} & =\text { inverse of a matrix }[*] \\ {[*]^{T}} & =\text { transpose of a matrix }[*]\end{array}$

\section{Introduction}

CLEXIBLE and light weight optical fibers not only revolutionize the telecommunications, but also innovate the sensing world. Optical fibers can be used as fiber optic sensors to measure strain, temperature, pressure, and other quantities. Properties of light, such as intensity, phase, transit time of light, et cetera, that propagate in the fiber can be altered by modifying a fiber. Recently, fiber optic sensors have been developed to measure co-located strain simultaneously with very high accuracy using fiber Bragg gratings (FBGs). Specifically, a fiber optic strain sensor (FOSS) uses a series of FBGs to obtain measurements at intervals as small as every millimeter ${ }^{1}$ along a fiber and a frequencies of several $\mathrm{kHz} .{ }^{2}$ The ability of FBGs to operate at such high frequencies makes them an ideal choice for both static and dynamic aerospace applications.

Obtaining wing deflections during flight are essential measurements for structural health monitoring and active flexible motion controls. Calculating wing deflection values from the measured FOSS data is available along the singular optical fibers. Ko et al. $^{3}$ uses closed-form equations to calculate real-time operational loads and deflections on complex structures using surface strain data. The Kang et al. ${ }^{4}$ calculation method uses a matrix equation based on

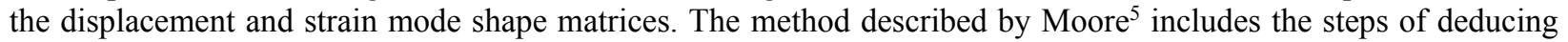
curvature and bend radius data for the optical fiber from the strain data: curve-fitting to obtain curvature and bending direction functions pertaining to the optical fiber, calculating a torsion function using the bending direction function, and calculating the three-dimensional shape of the optical fiber from the combination of the curvature, and bending and torsion functions. These three methods are suitable for calculating deflection and load values in cross-sectional lumps based on the location of the sensors used to measure strain on the surface.

The availability of wing deformation at all element grid points across the structural finite element (FE) model will improve the ability to monitor the health and behavior of a complex three dimensional structure such as an aircraft in flight. Several studies have been performed which evaluated using an inverse finite element method. ${ }^{6,7,8}$ This method is based on the minimization of a weighted-least-squares functional that uses the complete set of strain measurements. The two-step approach developed by $\mathrm{Pak}^{9}$ computes wing deflection values along the location of all the FOSS first, and then these deflection values are expanded to the entire structure using the System Equivalent Reduction and Expansion Process (SEREP) ${ }^{10}$ technique.

Acceleration and velocity of structural motion are also needed to compute internal and external loads during flight through the use of the structural governing equations of motion. This paper focuses on a computation of acceleration and velocity over the entire three dimensional structure based on measured strain information. First, unsteady structural deformation of the entire three dimensional structure is obtained using the two-step approach introduced by $\mathrm{Pak}^{9}$. Next, accelerations and velocities are computed using a simple harmonic motion assumption and a central difference equation, respectively.

\section{Mathematical Background}

A long term objective of the current study is the computation of external aerodynamic loads and internal structural loads from strains which are measured from a FOSS. This objective can be accomplished through the use of the structural dynamic governing equations of motion. Mass, damping, and stiffness matrices in Eq. (1) are from a finite element model. From Eq. (1), deflection, acceleration, and velocity values are needed to compute elastic, inertia, and damping loads. Therefore, computing deflection, acceleration, and velocity information from the measured strain are the critical quantities for the long term objectives. In the first section, deflections and slopes of an entire structure are calculated from measured strain through the use of a recently developed two-step theory. ${ }^{9}$ Acceleration and velocities are computed in the second and third sections using a harmonic motion assumption and the central difference equation, respectively.

\section{A. Computation of Wing Deflection from Measured Strain}

Consider the following structural dynamic governing equations of motion shown in Eq. (1):

$$
[\mathbf{M}]\{\ddot{\boldsymbol{q}}\}_{\boldsymbol{k}}+[\mathbf{G}]\{\dot{\boldsymbol{q}}\}_{\boldsymbol{k}}+[\mathbf{K}]\{\boldsymbol{q}\}_{k}=\left\{\boldsymbol{Q}_{\boldsymbol{a}}\right\}_{\boldsymbol{k}}
$$

where $[\mathbf{M}],[\mathbf{G}]$, and $[\mathbf{K}]$ are mass, damping, and stiffness matrices, respectively, and $\{\boldsymbol{q}\}_{\boldsymbol{k}}$ and $\left\{\boldsymbol{Q}_{\boldsymbol{a}}\right\}_{\boldsymbol{k}}$ are the generalized coordinates and the generalized aerodynamic load vectors at discrete time $k$, respectively. 
Deflections along the location of the FOSS can be computed from the measured strain data $\{\boldsymbol{\epsilon}\}_{\boldsymbol{k}}$ using a piecewise least-squares method, an Akima spline, and a linear assumption as described in the two-step approach. ${ }^{9}$ These computed deflections at discrete the FOSS locations are combined with a FE model of the structure in order to interpolate and extrapolate the deflection and slope of the entire structure through the use of SEREP. Re-arranging all the degrees of freedom (DOF) in the FE model yields Eq. (2):

$$
\{\boldsymbol{q}\}_{k}=\left\{\begin{array}{l}
\boldsymbol{q}_{M} \\
\boldsymbol{q}_{S}
\end{array}\right\}_{k}=[\boldsymbol{\Phi}]\{\boldsymbol{\eta}\}_{k}=\left[\begin{array}{l}
\boldsymbol{\Phi}_{M} \\
\boldsymbol{\Phi}_{S}
\end{array}\right]\{\boldsymbol{\eta}\}_{k}
$$

where $\left\{\boldsymbol{q}_{\boldsymbol{M}}\right\}_{\boldsymbol{k}}$ is the master DOF at discrete time $k$. In this approach, deflections along the fiber locations computed from the first step of the two-step approach ${ }^{9}$ are defined as the master DOF. The rest of the deflections and slopes all over the structure are defined as slave DOF at discrete time $k,\left\{\boldsymbol{q}_{s}\right\}_{\boldsymbol{k}}$. In Eq. (2), matrices $\left[\boldsymbol{\Phi}_{\boldsymbol{M}}\right]$ and $\left[\boldsymbol{\Phi}_{s}\right]$ are eigen-matrices corresponding to master and slave degrees of freedom, respectively, and $\{\boldsymbol{\eta}\}_{\boldsymbol{k}}$ is the orthonormalized coordinates vector at discrete time $k$. Therefore, the following two equations, Eqs. (3) and (4), are derived from Eq. (2).

$$
\begin{aligned}
\left\{\boldsymbol{q}_{\boldsymbol{M}}\right\}_{\boldsymbol{k}} & =\left[\boldsymbol{\Phi}_{\boldsymbol{M}}\right]\{\boldsymbol{\eta}\}_{\boldsymbol{k}} \\
\left\{\boldsymbol{q}_{S}\right\}_{\boldsymbol{k}} & =\left[\boldsymbol{\Phi}_{S}\right]\{\boldsymbol{\eta}\}_{\boldsymbol{k}}
\end{aligned}
$$

In Eq. (3), changing master DOF at discrete time $k,\left\{\boldsymbol{q}_{\boldsymbol{M}}\right\}_{\boldsymbol{k}}$ to the corresponding measured values $\left\{\widetilde{\boldsymbol{q}}_{\boldsymbol{M}}\right\}_{\boldsymbol{k}}$ gives Eq. (5):

$$
\left\{\widetilde{\boldsymbol{q}}_{M}\right\}_{\boldsymbol{k}}=\left[\boldsymbol{\Phi}_{M}\right]\{\boldsymbol{\eta}\}_{\boldsymbol{k}}
$$

where $\left\{\widetilde{\boldsymbol{q}}_{\boldsymbol{M}}\right\}_{\boldsymbol{k}}$ is obtained from the first step of the two-step approach. ${ }^{9}$ Pre-multiply $\left[\boldsymbol{\Phi}_{\boldsymbol{M}}\right]^{T}$ to Eq. (5) with matrix inversion gives Eq. (6) for computing the orthonormalized coordinates vector at discrete time $k$,

$$
\{\boldsymbol{\eta}\}_{k}=\left(\left[\boldsymbol{\Phi}_{M}\right]^{T}\left[\boldsymbol{\Phi}_{M}\right]\right)^{-1}\left[\boldsymbol{\Phi}_{M}\right]^{T}\left\{\widetilde{\boldsymbol{q}}_{M}\right\}_{k},
$$

and the generalized coordinates vector $\{\boldsymbol{q}\}_{\boldsymbol{k}}$ is obtained from Eq. (2).

\section{B. Computation of Acceleration from Computed Wing Deflection and Frequencies}

From Eq. (2), acceleration at discrete time $k$ can be expressed as shown in Eq. (7).

$$
\{\ddot{\boldsymbol{q}}\}_{k}=\left\{\begin{array}{l}
\ddot{\boldsymbol{q}}_{M} \\
\ddot{\boldsymbol{q}}_{S}
\end{array}\right\}_{k}=\left[\begin{array}{l}
\boldsymbol{\Phi}_{M} \\
\boldsymbol{\Phi}_{S}
\end{array}\right]\{\ddot{\boldsymbol{\eta}}\}_{k}
$$

By assuming simple harmonic motion for the i-th orthonormalized coordinate, $\ddot{\eta}_{i}(k)=-\omega_{i}^{2} \eta_{i}(k) i=1,2, \ldots, n$, acceleration can be obtained from deflections and frequencies as shown in Eq. (8).

$$
\{\ddot{\boldsymbol{\eta}}\}_{\boldsymbol{k}}=\left\{\begin{array}{c}
\ddot{\eta}_{1}(k) \\
\ddot{\eta}_{2}(k) \\
\vdots \\
\ddot{\eta}_{n}(k)
\end{array}\right\}=-\left[\begin{array}{cccc}
\omega_{1}^{2} & 0 & \ldots & 0 \\
0 & \omega_{2}^{2} & \ldots & 0 \\
0 & 0 & \ddots & \vdots \\
0 & 0 & \ldots & \omega_{n}^{2}
\end{array}\right]\left\{\begin{array}{c}
\eta_{1}(k) \\
\eta_{2}(k) \\
\vdots \\
\eta_{n}(k)
\end{array}\right\}=-\left[\boldsymbol{\omega}_{\boldsymbol{i}}^{2}\right]\{\boldsymbol{\eta}\}_{\boldsymbol{k}}
$$

Substituting Eq. (8) into Eq. (7) gives Eq. (9).

$$
\{\ddot{\boldsymbol{q}}\}_{k}=-\left[\begin{array}{l}
\boldsymbol{\Phi}_{M}\left[\boldsymbol{\omega}_{i}^{2}\right] \\
\boldsymbol{\Phi}_{S}\left[\boldsymbol{\omega}_{i}^{2}\right]
\end{array}\right]\{\boldsymbol{\eta}\}_{k}
$$

Therefore, acceleration is computed using Eqs. (6) and (9). In Eq. (8), frequencies $\omega_{i} i=1,2, \ldots, n$, are computed from unsteady strain distribution at a selected point using an on-line parameter estimation technique together with an autoregressive moving average (ARMA) model. ${ }^{11}$ 


\section{Computation of Velocity from Computed Wing Deflection and Frequencies}

From Eq. (2), velocity at time $k$ can be written as shown in Eq. (10).

$$
\{\dot{\boldsymbol{q}}\}_{\boldsymbol{k}}=\left\{\begin{array}{l}
\dot{\boldsymbol{q}}_{M} \\
\dot{\boldsymbol{q}}_{S}
\end{array}\right\}_{\boldsymbol{k}}=\left[\begin{array}{c}
\boldsymbol{\Phi}_{M} \\
\boldsymbol{\Phi}_{S}
\end{array}\right]\{\dot{\boldsymbol{\eta}}\}_{\boldsymbol{k}}
$$

Consider the following central difference equation, Eq. (11), for the time derivative of orthonormalized coordinates vector $\{\dot{\boldsymbol{\eta}}\}_{k}$.

$$
\{\dot{\boldsymbol{\eta}}\}_{\boldsymbol{k}}=\frac{\{\boldsymbol{\eta}\}_{k+1}-\{\boldsymbol{\eta}\}_{k-1}}{2 \Delta t}
$$

The time derivative of the i-th orthonormalized coordinate can be written in Eq. (12).

$$
\dot{\eta}_{i}(k)=\frac{\eta_{i}(k+1)-\eta_{i}(k-1)}{2 \Delta t}
$$

It should be noted in Eqs. (11) and (12) that the orthonormalized coordinates vector at future time $k+1$ is needed to have the time derivative of the orthonormalized coordinates vector at time $k$.

Consider a linear autoregressive (AR) model $^{11}$ for the i-th orthonormalized coordinate $\eta_{i}(k)$ given in Eq.(13):

$$
\eta_{i}(k)=a_{1 i} \eta_{i}(k-1)+a_{2 i} \eta_{i}(k-2)
$$

where AR coefficients for the $\mathrm{i}$-th orthonormalized coordinate, $a_{1 i}$ and $a_{2 i}$, are computed from the same i-th frequency $\omega_{i}$ in Eq.(8). From Eq. (13), the i-th orthonormalized coordinate at future time $k+1$ can be expressed as shown in Eq.(14).

$$
\eta_{i}(k+1)=a_{1 i} \eta_{i}(k)+a_{2 i} \eta_{i}(k-1)
$$

Substituting Eq. (14) into Eq. (12) gives Eq.(15).

$$
\dot{\eta}_{i}(k)=\frac{a_{1 i} \eta_{i}(k)+\left(a_{2 i}-1\right) \eta_{i}(k-1)}{2 \Delta t}
$$

In summary, velocity of a flexible wing motion is computed as follows:

Step 1: compute the orthonormalized coordinates vector $\{\boldsymbol{\eta}\}_{\boldsymbol{k}}$ from Eq. (6).

Step 2: compute the time derivative of the orthonormalized coordinates vector $\{\dot{\boldsymbol{\eta}}\}_{\boldsymbol{k}}$ from Eqs. (15) and (16).

$$
\{\dot{\boldsymbol{\eta}}\}_{\boldsymbol{k}}=\left\{\begin{array}{c}
\dot{\eta}_{1}(k) \\
\dot{\eta}_{2}(k) \\
\vdots \\
\dot{\eta}_{i}(k)
\end{array}\right\}
$$

Step 3: compute the time derivative of the generalized coordinates vector $\{\dot{\boldsymbol{q}}\}_{\boldsymbol{k}}$ from Eq. (10).

Therefore, deflection, acceleration, and velocity based on the generalized coordinates vectors $\{\boldsymbol{q}\}_{\boldsymbol{k}},\{\ddot{\boldsymbol{q}}\}_{\boldsymbol{k}}$, and $\{\dot{\boldsymbol{q}}\}_{\boldsymbol{k}}$, as well as the orthonormalized coordinates vectors $\{\boldsymbol{\eta}\}_{k},\{\ddot{\boldsymbol{\eta}}\}_{\boldsymbol{k}}$, and $\{\dot{\boldsymbol{\eta}}\}_{\boldsymbol{k}}$, are computed from the measured strain data $\{\boldsymbol{\epsilon}\}_{\boldsymbol{k}}$. 


\section{Results and Discussions}

The theory presented in this study is validated using a cantilevered rectangular wing model as shown in Fig. 1. This FE model represents a wind tunnel test article ${ }^{12}$ that has a 0.065 -inch thick aluminum plate with a thin plastic foam skin that forms a $6 \%$ circular arc cross-sectional shape. A transient load using a half sine short duration pulse is applied at the tip leading edge corner. Strain values are output from each zero stiffness beam element segment at simulated FOSS fiber locations along the span, a total of 50 beam elements for each fiber and along upper and lower plate elements as shown in Fig. 2. The upper and lower elements are offset from the center plate elements to generate non-zero strain. Note that these elements have a finer distribution at the root as required to lower the propagation of integration errors outwards to the tip.

For validation and refinement of this theory, a computational validation was performed. This wing model is analyzed using the MSC/NASTRAN. ${ }^{13}$ The computed strain values are assumed to be strain output from a FOSS. The computed deflection, acceleration, and velocity values are considered as the targets to be compared with the results obtained from the current approach.

The cantilevered rectangular wing FE model has two versions that differ in material and mass properties to test the current approach with a real world variation of model accuracy as compared to measured frequency results. These two FE model versions are shown in Table 1 where case 1 has its plastic foam mass equally smeared among the aluminum plate density, and case 2 has the plastic foam mass distributed as lumped masses over the $6 \%$ circular-arc cross sectional shape. Young's modulus and shear modulus for the case 2 model are tuned using the structural dynamic model tuning tool developed at the National Aeronautics and Space Administration (NASA) Armstrong Flight Research Center. ${ }^{14}$ In Table 2, computed modal frequencies for cases 1 and 2 are compared to available measured values. ${ }^{12}$ The case 1 frequencies have up to a $5.6 \%$ error which is typical of such FE models near the limits of the 5\% frequency NASA FE modeling standard. ${ }^{15}$ The frequency errors for case 2 , at no more than $0.8 \%$, represent an FE model that has the best accuracy that can usually be expected.

Both cases 1 and 2 were run to generate the target responses with NASTRAN modal transient response analysis (sol 112) with 1200 time steps. The case 1 mode shapes are used to calculate transformation matrices for both cases. The case 1 mode shapes are the eigen and comparison functions for the case 1 and case 2 transient responses, respectively.

Frequency estimations are generated using computed strain values for cases 1 and 2 and are taken at the leading edge root as shown in Fig. 2. The computed strain time histories for both cases are shown in Fig. 3. The frequency estimation uses Bierman's U-D factorization algorithm with 20 AR coefficients, a covariance matrix resetting interval of $80 \mathrm{steps}$, and a forgetting factor of 0.98 . For cases 1 and 2, sampling times of $0.0006267 \mathrm{~s}$ and $0.0006487 \mathrm{~s}$, and Nyquist frequencies of $797.9 \mathrm{~Hz}$ and $770.8 \mathrm{~Hz}$, respectively, were used. These estimated frequencies are compared to the target values generated from the NASTRAN output shown in Table 3. The estimated frequency errors are very low, if not at zero, with the highest standing out at $-0.09 \%$ for case 2 , mode 1 .

Using the current approach, deflections and pitch angles are calculated using computed strain along simulated fiber locations of the wing model. For case 1, target deflection, acceleration, and velocity values are compared to the current approach calculations using 22 fiber locations at the wing model leading edge tip grid 51, shown in Fig. 2. Since grid 51 is located on the leading edge fiber, its deflections are directly computed from strain values and are master DOF. Using wider and close up time histories, the out of plane, or $\mathrm{z}$ deflections are shown in Fig. 4, and the pitch angles are shown in Fig. 5. The green dot line and the black solid line represent the target and values from the current method, respectively. The $\mathrm{z}$ deflection comparisons are so close to each other that no discernable differences can be seen in the plots between the target and current method values. The pitch angle comparisons are almost as equally indiscernible from each other except at several of the areas with large changes in gradient where, in general, the target values are a little larger than those for the current method.

In Fig. 4(a), the period of the large oscillation is approximately $0.065 \mathrm{~s}, 15.4 \mathrm{~Hz}$. Therefore, the first natural frequency is observed in the $\mathrm{z}$ deflection response. On the other hand, seven oscillations are observed for $0.09 \mathrm{~s}$ in Fig. 5(a). Therefore, the period of the large oscillation is approximately $0.0129 \mathrm{~s}, 77.8 \mathrm{~Hz}$. This frequency corresponds to the second mode, $77.4 \mathrm{~Hz}$, and is the first torsion mode. In Fig. 5(b), the small oscillation is also dominant, and three oscillations are observed during $0.0041 \mathrm{~s}$, and therefore the corresponding frequency is approximately $730 \mathrm{~Hz}$, period is $0.00137 \mathrm{~s}$.

Out of plane, or z direction, accelerations are compared in Fig. 6, and comparisons for pitch accelerations are shown in Fig. 7. Both acceleration plots are showing an initial opposite trend in phase between the target and current method values for about a half cycle, but the current method very quickly recovers and begins to match up closely. This initial phase difference is mainly due to the simple harmonic assumption for the transient responses. For the $\mathrm{z}$ accelerations, the comparisons are showing several peak values with slightly larger target values over those for the

5

American Institute of Aeronautics and Astronautics 
current method. The pitch acceleration comparisons are showing the same match of trend with more of the target peak values showing some larger values over those for the current method.

Out of plane velocity and pitch rate based on the current method values are compared with the corresponding target responses in Figs. 8 and 9, respectively. Both figures show that the current method values are smaller than the target values during the transient time period. Slightly larger target values over the corresponding values for the current method are also observed in the $\mathrm{z}$ velocity and pitch rate responses.

The case 2 wing model comparisons of the target results to the current method using 22 fiber locations are made at a tip 1/3 aft chord location, grid point 2601 as shown in Fig. 2. The $\mathrm{z}$ deflections and pitch angle time histories are shown in Figs.10 and 11, respectively. The matching and small deviations of the target values to those of the current method are essentially the same as they were for case 1, for both the $\mathrm{z}$ deflections and the pitch angles. In Fig. 10(a), the period of the large oscillation is approximately $0.068 \mathrm{~s}, 14.7 \mathrm{~Hz}$. Similar to case 1, the first natural frequency $14.29 \mathrm{~Hz}$ is observed in the $\mathrm{z}$ deflection response. On the other hand, eight oscillations are observed for $0.10 \mathrm{~s}$ in Fig. 11(a), at approximately $80 \mathrm{~Hz}$. Therefore, the $80.18 \mathrm{~Hz}$ torsion mode is dominant in pitch motion.

The $\mathrm{z}$ and pitch accelerations time history comparisons are shown in Figs. 12 and 13, respectively. The $\mathrm{z}$ velocity and the pitch rate comparisons are given in Figs. 14 and 15, respectively. And again, these results are essentially repeating the same trends as seen with case 1 .

The target to the current approach results for the wing model cases 1 and 2 are summarized in Table 4 . An additional sensitivity study of a fewer number of fibers for case 2 is also shown in Table 4 . The previous number of fibers used for case 2 is lowered from 22 to 10 and 6, and is depicted in Fig. 16. Percent errors between the target and current method results are calculated using Eq. (17).

$$
\% \text { Error } \equiv \frac{\sum_{k=0}^{n} \mid \text { Current } \operatorname{approach}(k)-\operatorname{Target}(k) \mid}{\sum_{k=0}^{n}|\operatorname{Target}(k)|}
$$

The target values are those directly computed from the FE wing model. The results at discrete time, $k$ are summed for all the time histories.

Table 4 is showing a very small general increase in error with a reduction of the number of fibers for case 2 . This minimal increase in error is an indication of the robustness of the least-squares surface fitting procedure from the fiber locations out to the rest of the wing model. For case 1, with the $\mathrm{z}$ and pitch accelerations at grid 51 and on a fiber location, the errors in $\mathrm{z}$ acceleration are noticeably lower than those at an off fiber location at grid 2601. It should be noted that the $\mathrm{z}$ deflection at grid point 51 belongs to the master DOF. However, any DOF at grid point 2601 belong to the slave DOF. The $\mathrm{z}$ deflection results have the lowest percent error, at no more than $1.79 \%$. The pitch angles, $\mathrm{z}$ accelerations, pitch accelerations, $\mathrm{z}$ velocity, and pitch rate calculated during and after the second step, as slave DOF; have higher errors are higher at $6.35 \%, 17.6 \%, 10.2 \%, 19.0 \%$, and $12.0 \%$, respectively. It should also be emphasized that the comparison functions are used for case 2 instead of using eigen functions. However, the quality of the results are not degraded too much.

Finally, instead of using the central difference equation, Eq. (11), the following backward difference equation given in Eq. (18) is used for the pitch rate computations with the case 2 model.

$$
\{\dot{\boldsymbol{\eta}}\}_{k}=\frac{\{\boldsymbol{\eta}\}_{\boldsymbol{k}}-\{\boldsymbol{\eta}\}_{\boldsymbol{k}-\mathbf{1}}}{\Delta t}
$$

Comparisons between the central difference equation with a linear AR model and the backward difference equation are shown in Fig. 17. In Fig. 17(b), small phase differences are observed between the target responses and the values from the current study. It should be concluded that the current proposed central difference equation with a linear AR model closely follows the target response.

\section{Conclusion}

A simple approach for computing acceleration and velocity of a structure from the time histories of strain is proposed in this study. First, deflection and slope of the structure are computed from strain using a two-step theory. Next, the simple harmonic motion assumption is used for the acceleration computations. Therefore, accelerations are computed from the deflections obtained from the first step of the two-step theory and the estimated frequencies. Frequencies of the structure are computed from the time histories of strain using a parameter estimation technique together with an autoregressive moving average model. Finally, the central difference equation with a linear AR model 
is used for the computations of velocity. A cantilevered rectangular wing model is used to validate the simple approach.

Quality of the computed deflection, acceleration, and velocity values are independent of the number of fibers (number of master DOF) except using too small of a number. If only a single fiber is used for the whole surface, then the torsional motion is not captured properly.

Velocity values based on the central difference equation with a linear AR model proposed in this study follows the target response with reasonable accuracy. Therefore, the phase shift issue associated with the backward difference equation is successfully overcome using the central difference equation with a linear AR model proposed in this study.

A future application of the approach will be performed on the X-56A aeroelastic research aircraft (Lockheed Martin, Bethesda, Maryland) in order to generate pre-flight test estimates of aerodynamic forces on the wing surfaces. These force estimates will be correlated to those results as derived from onboard FOSS strain flight data. 
Tables

Table 1. Wing finite element model properties.

\begin{tabular}{|c|c|c|}
\hline \hline Properties & Case 1 model & Case 2 model \\
\hline Young's modulus & $9847900 \mathrm{psi}$ & $\mathbf{9 2 0 7 7 6 6} \mathrm{psi}$ (tuned value) \\
\hline Shear modulus & $3639672 \mathrm{psi}$ & $\mathbf{3 8 3 6 5 7 0} \mathrm{psi}$ (tuned value) \\
\hline Density & $0.11166 \mathrm{lb} / \mathrm{inch}^{3}$ & $0.1 \mathrm{lb} /$ inch $^{3}$ \\
\hline Foam weight & Smeared & Lumped mass \\
\hline Total weight & $0.3806 \mathrm{lb}$ & $0.3806 \mathrm{lb}$ \\
\hline Xcg & $2.28 \mathrm{inch}$ & $2.28 \mathrm{inch}$ \\
\hline Ycg & $5.75 \mathrm{inch}$ & $5.75 \mathrm{inch}$ \\
\hline Thickness & $0.065 \mathrm{inch}$ & $0.065 \mathrm{inch}$ \\
\hline \hline
\end{tabular}

Table 2. Measured versus computed frequencies.

\begin{tabular}{|c|c|c|c|c|c|}
\hline \hline Mode & Measured $^{\mathbf{1 2}}(\mathbf{H z})$ & Case 1 (Hz) & $\mathbf{\% ~ E r r o r ~}$ & Case 2 (Hz) & \% Error \\
\hline 1 & 14.29 & 15.09 & $\mathbf{5 . 6}$ & 14.29 & $\mathbf{0 . 0}$ \\
\hline 2 & 80.41 & 77.40 & $\mathbf{- 3 . 7}$ & 80.17 & $\mathbf{- 0 . 3}$ \\
\hline 3 & 89.80 & 93.57 & $\mathbf{4 . 2}$ & 89.04 & $\mathbf{- 0 . 8}$ \\
\hline 4 & N/A & 246.37 & N/A & 248.76 & N/A \\
\hline 5 & N/A & 262.02 & N/A & 252.41 & N/A \\
\hline 6 & N/A & 455.22 & N/A & 459.34 & N/A \\
\hline 7 & N/A & 511.27 & N/A & 485.61 & N/A \\
\hline 8 & N/A & 642.72 & N/A & 606.65 & N/A \\
\hline 9 & N/A & 722.32 & N/A & 718.59 & N/A \\
\hline 10 & N/A & 773.93 & N/A & 747.65 & N/A \\
\hline
\end{tabular}

Table 3. Estimated system frequencies for cases 1 and 2.

\begin{tabular}{|c|c|c|c|c|c|c|}
\hline \hline \multirow{2}{*}{ Mode Case 1 } & \multicolumn{3}{c|}{ Case 2 } \\
\cline { 2 - 7 } & Target (Hz) & Estimated (Hz) & \% Error & Target (Hz) & Estimated (Hz) & \% Error \\
\hline 1 & 15.09 & 15.09 & 0.00 & 14.29 & 14.28 & -0.09 \\
\hline 2 & 77.40 & 77.40 & 0.00 & 80.17 & 80.18 & 0.02 \\
\hline 3 & 93.57 & 93.57 & 0.00 & 89.04 & 89.05 & 0.01 \\
\hline 4 & 246.37 & 246.37 & 0.00 & 248.76 & 248.77 & 0.00 \\
\hline 5 & 262.02 & 262.02 & 0.00 & 252.41 & 252.41 & 0.00 \\
\hline 6 & 455.22 & 455.22 & 0.00 & 459.34 & 459.34 & 0.00 \\
\hline 7 & 511.27 & 511.27 & 0.00 & 485.61 & 485.61 & 0.00 \\
\hline 8 & 642.72 & 642.72 & 0.00 & 606.65 & 606.65 & 0.00 \\
\hline 9 & 722.32 & 722.32 & 0.00 & 718.59 & 718.60 & 0.00 \\
\hline 10 & 773.93 & 773.93 & 0.00 & 747.65 & 747.66 & 0.00 \\
\hline \hline
\end{tabular}

Table 4. Target and current method errors for case 1 and 2 simulations.

\begin{tabular}{|l|c|c|c|c|c|c|c|}
\hline \multirow{3}{*}{ Model } & \multirow{2}{*}{ Grid (\# of fiber) } & \multicolumn{9}{|c|}{ \% Error } \\
\cline { 3 - 7 } & & \multicolumn{2}{|c|}{ Deflection } & \multicolumn{2}{c|}{ Acceleration } & \multicolumn{2}{c|}{ Velocity } \\
\cline { 2 - 7 } & & $\mathbf{Z}$ & Pitch & $\mathbf{Z}$ & Pitch & $\mathbf{Z}$ & Pitch \\
\hline Case 1 & $51(22)$ & 1.55 & 5.36 & 6.42 & 7.96 & 10.5 & 12.0 \\
\hline \multirow{3}{*}{ Case 2 } & $2601(22)$ & 1.38 & 5.76 & 16.9 & 9.84 & 15.0 & 11.4 \\
\cline { 2 - 8 } & $2601(10)$ & 1.67 & 5.99 & 17.0 & 10.2 & 15.9 & 11.7 \\
\cline { 2 - 8 } & $2601(6)$ & 1.79 & 6.35 & 17.6 & 10.2 & 19.0 & 11.8 \\
\hline \hline
\end{tabular}

8

American Institute of Aeronautics and Astronautics 


\section{Figures}

22 Simulated FOSS locations using beam elements Strain data is taken at each of the $\mathbf{5 0}$ beam elements per fiber.

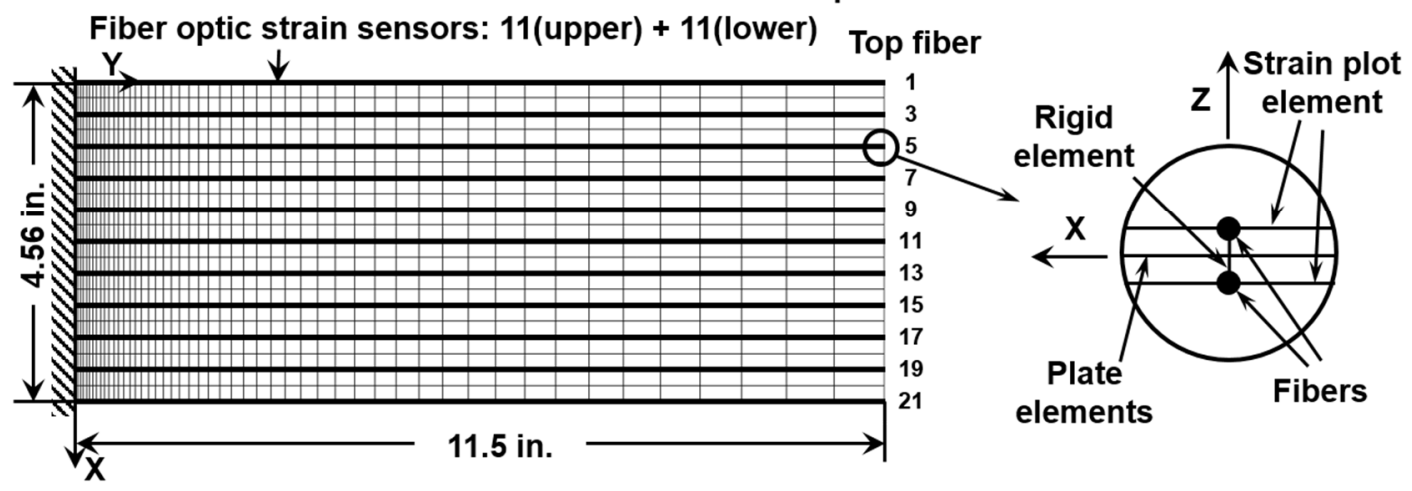

Figure 1. Cantilevered rectangular wing model.

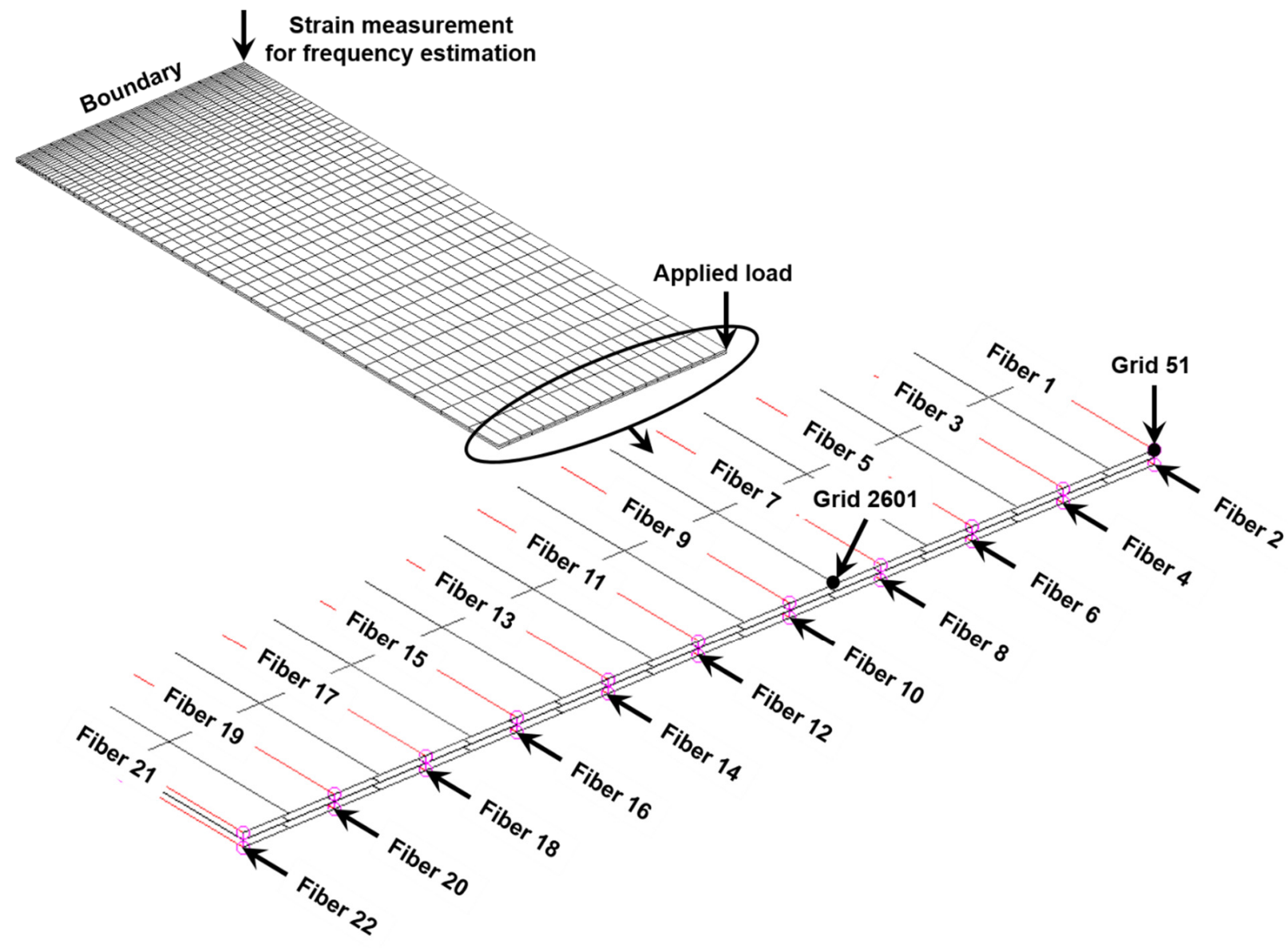

Figure 2. Wing model with simulated FOSS locations. 


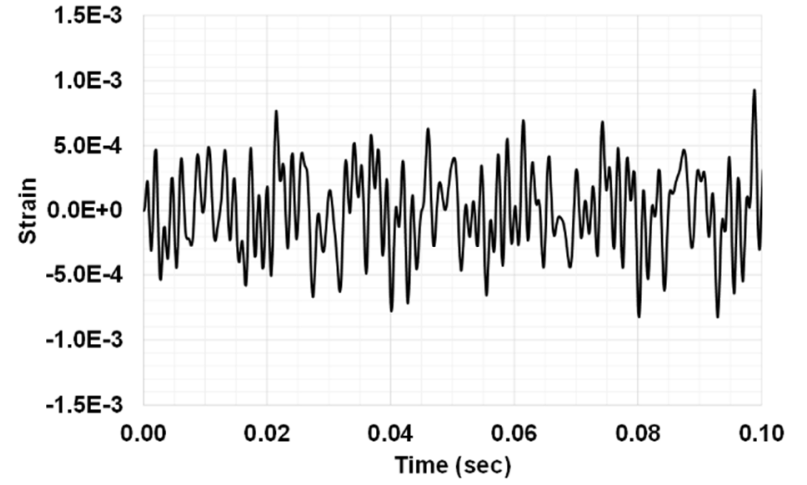

a) Case 1 .

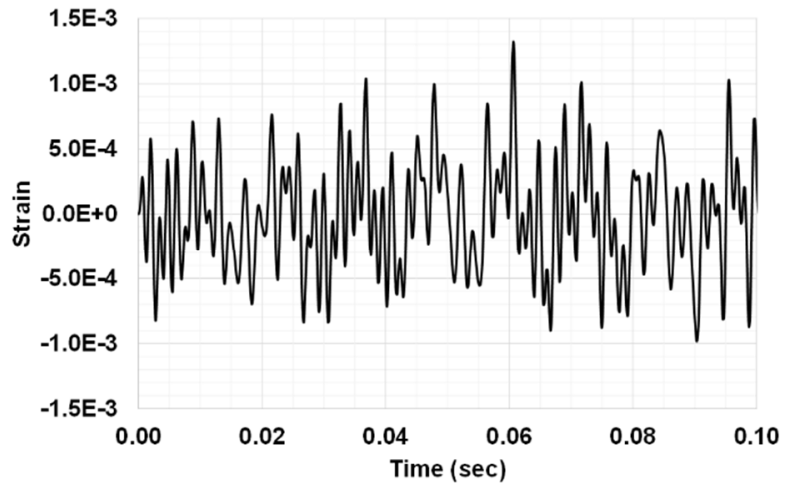

b) Case 2.

Figure 3. Strain time history at the leading edge of the wing root section for case 1 and case 2.

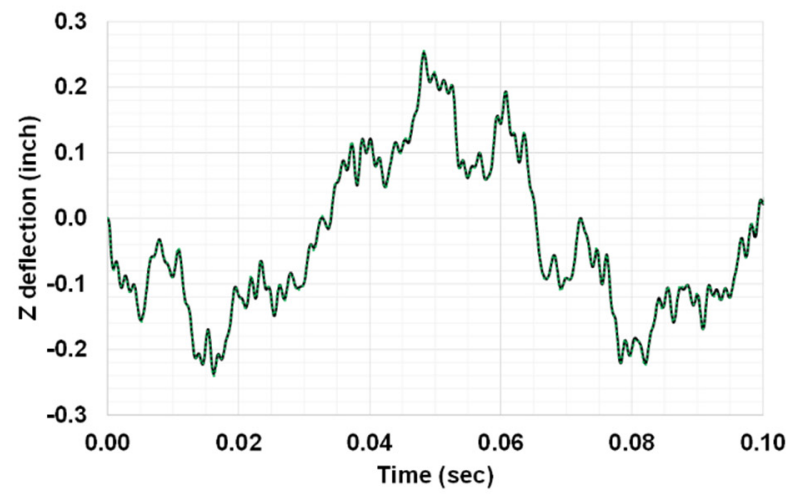

a) From 0 to $0.10 \mathrm{~s}$.

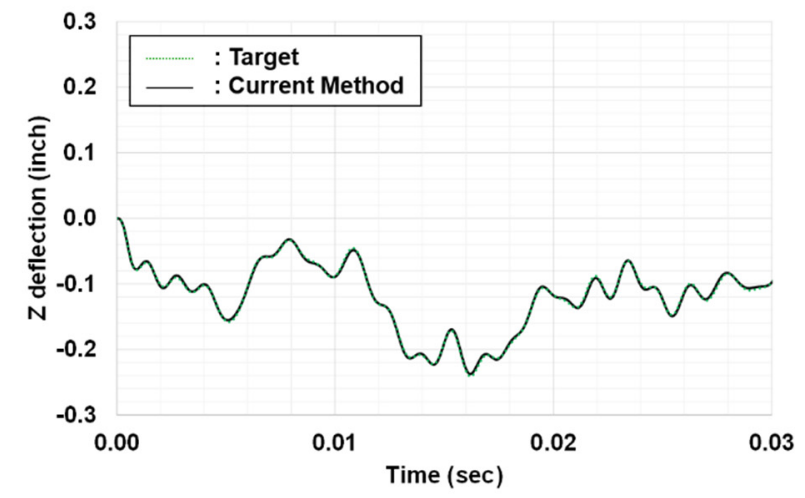

b) Zoom in from 0 to $0.03 \mathrm{~s}$.

Figure 4. Case 1, $\mathrm{Z}$ deflection time history at grid 51.

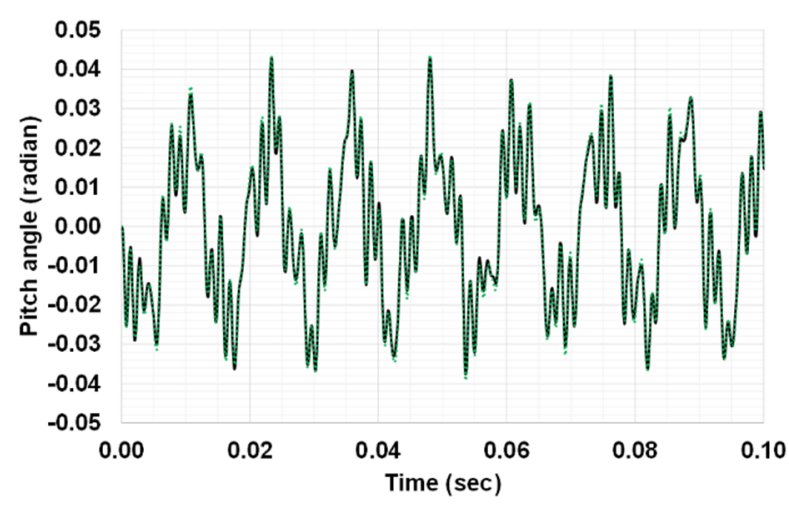

a) From 0 to $0.10 \mathrm{~s}$.

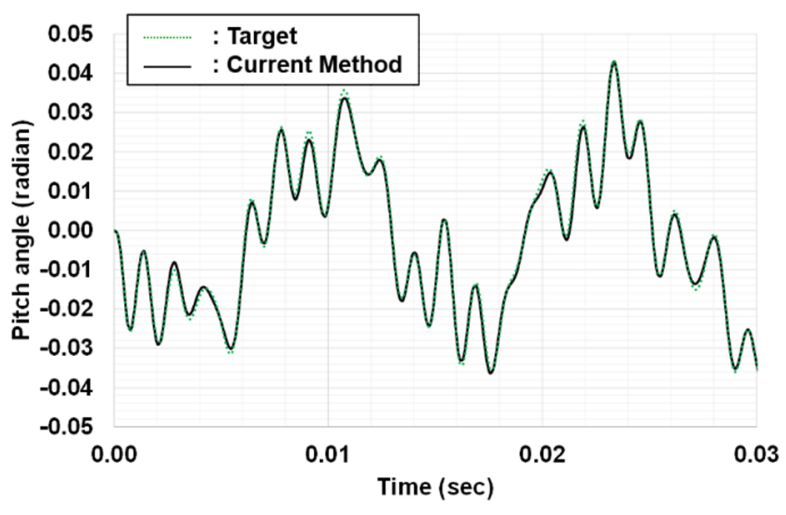

b) Zoom in from 0 to $0.03 \mathrm{~s}$.

Figure 5. Case 1, pitch angle time history at grid 51. 


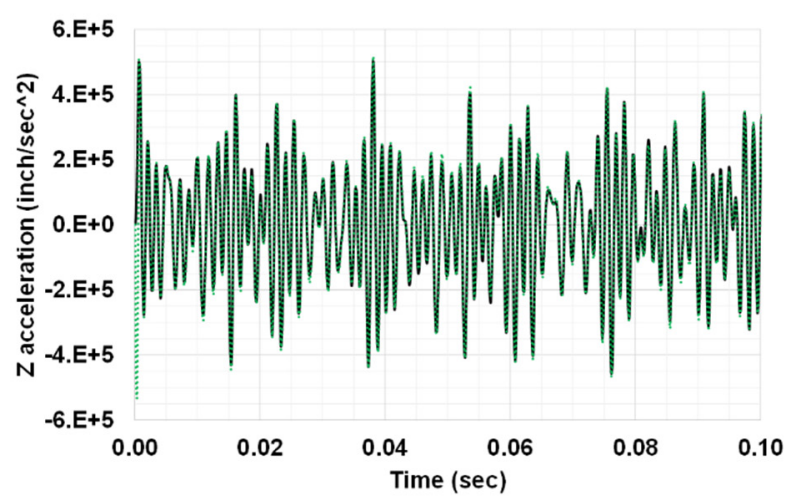

a) From 0 to $0.10 \mathrm{~s}$.

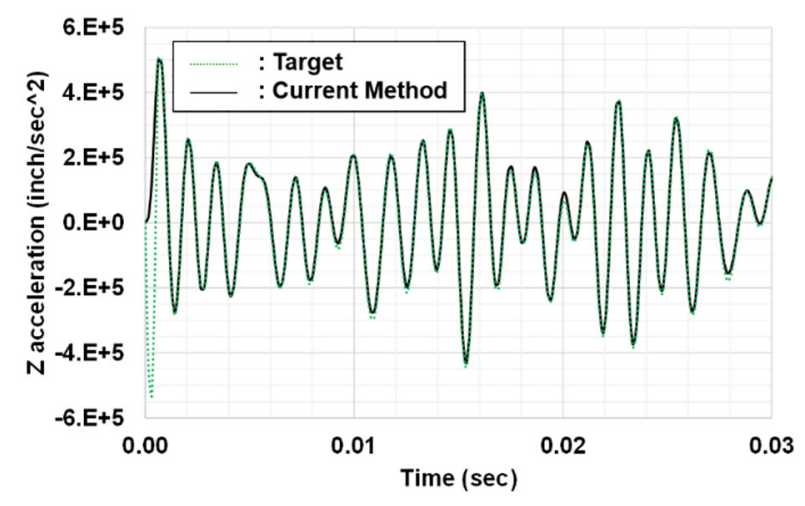

b) Zoom in from 0 to $0.03 \mathrm{~s}$..

Figure 6. Case 1, $\mathrm{Z}$ acceleration time history at grid 51.

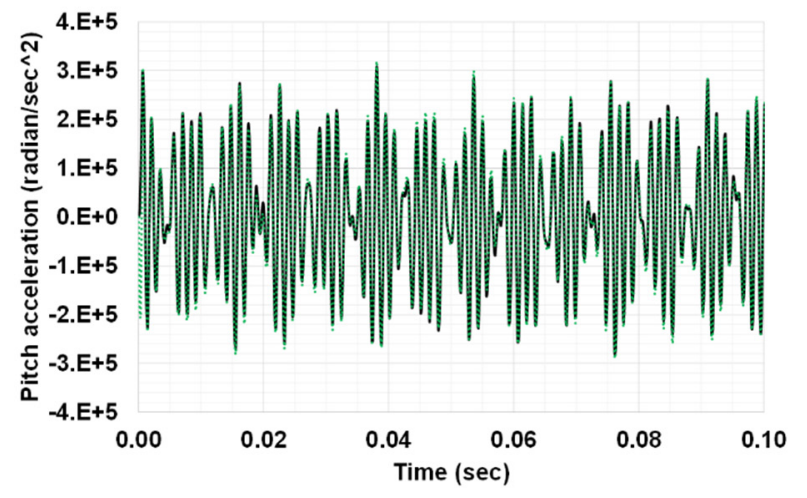

a) From 0 to $0.10 \mathrm{~s}$.

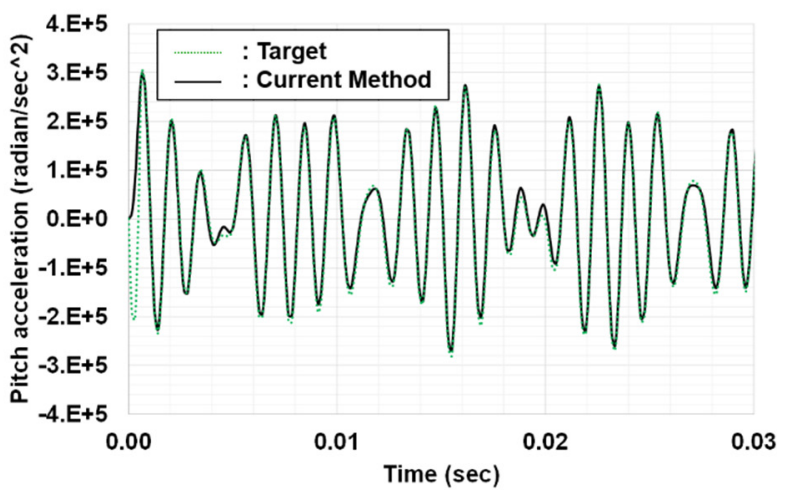

b) Zoom in from 0 to $0.03 \mathrm{~s}$.

Figure 7. Case 1, pitch acceleration time history at grid 51.

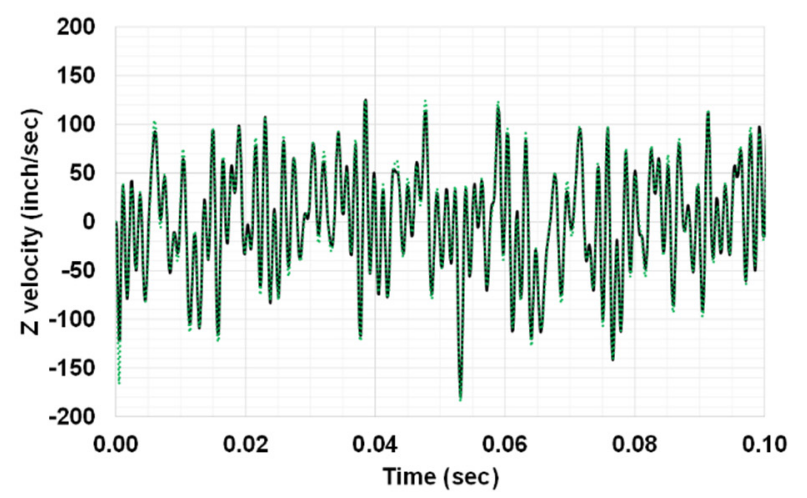

a) From 0 to $0.10 \mathrm{~s}$.

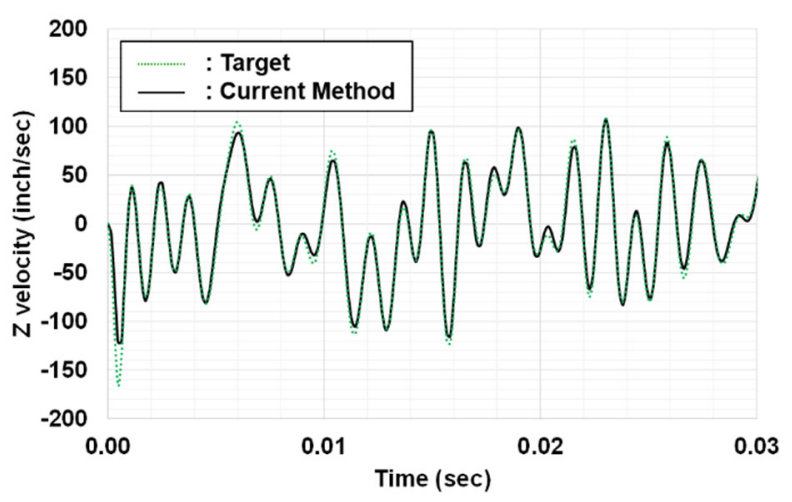

b) Zoom in from 0 to $0.03 \mathrm{~s}$.

Figure 8. Case 1, $\mathrm{Z}$ velocity time history at grid 51. 


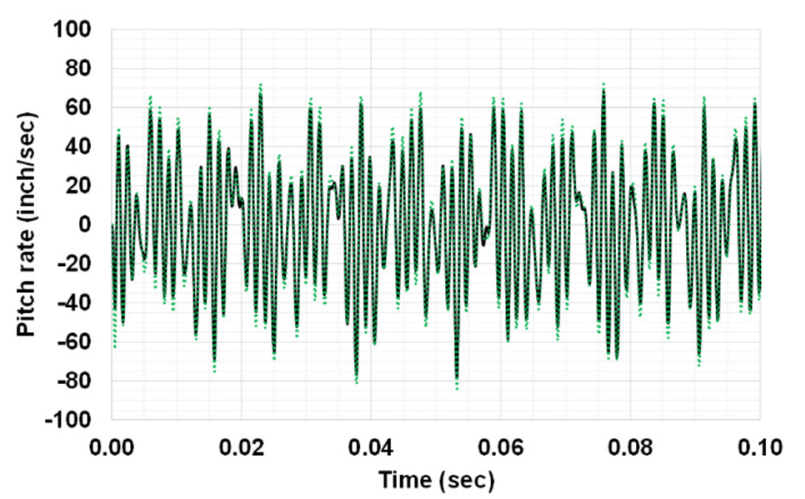

a) From 0 to $0.10 \mathrm{~s}$.

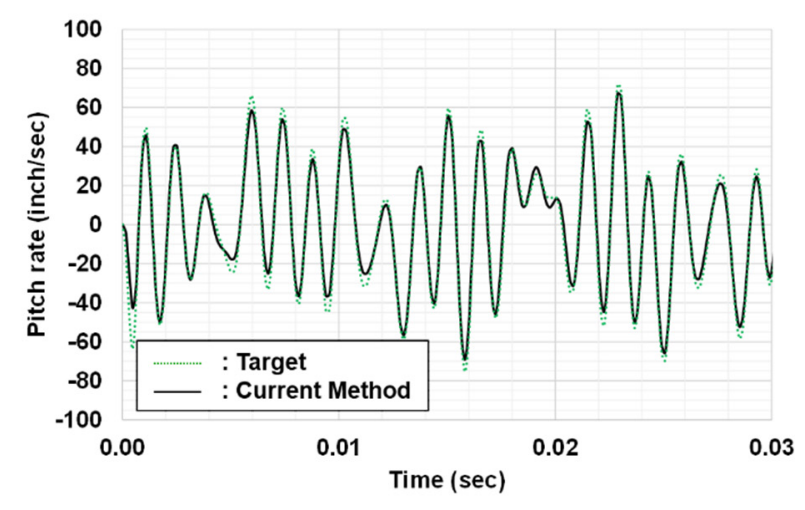

b) Zoom in from 0 to $0.03 \mathrm{~s}$.

Figure 9. Case 1, pitch rate time history at grid 51.

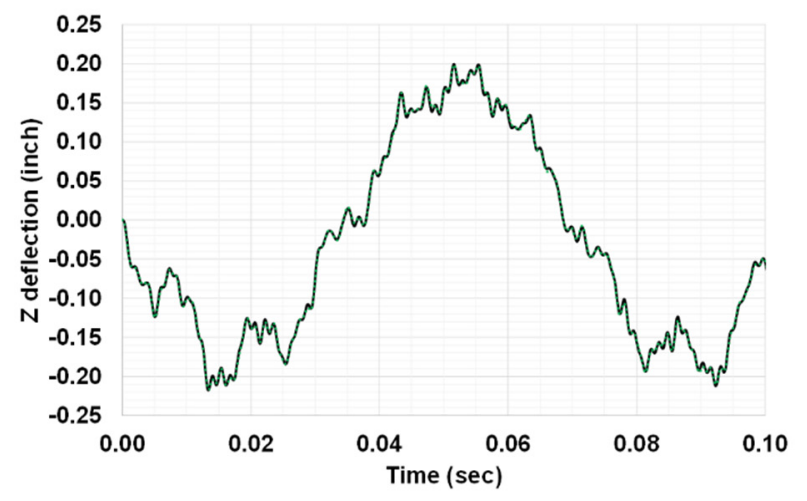

a) From 0 to $0.10 \mathrm{~s}$.

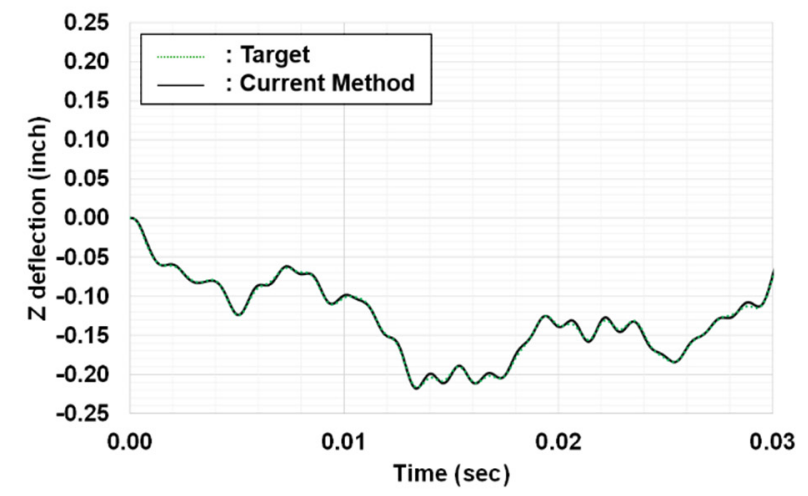

b) Zoom in from 0 to $0.03 \mathrm{~s}$.

Figure 10. Case 2, $\mathrm{Z}$ deflection time history at grid 2601.

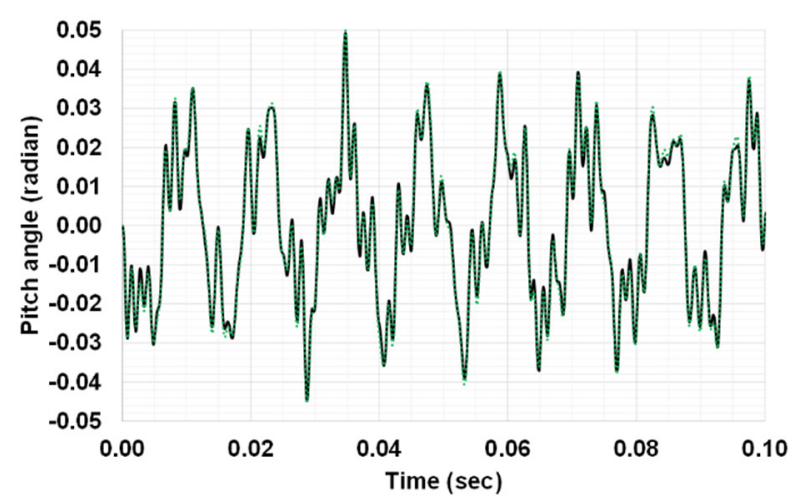

a) From 0 to $0.10 \mathrm{~s}$.

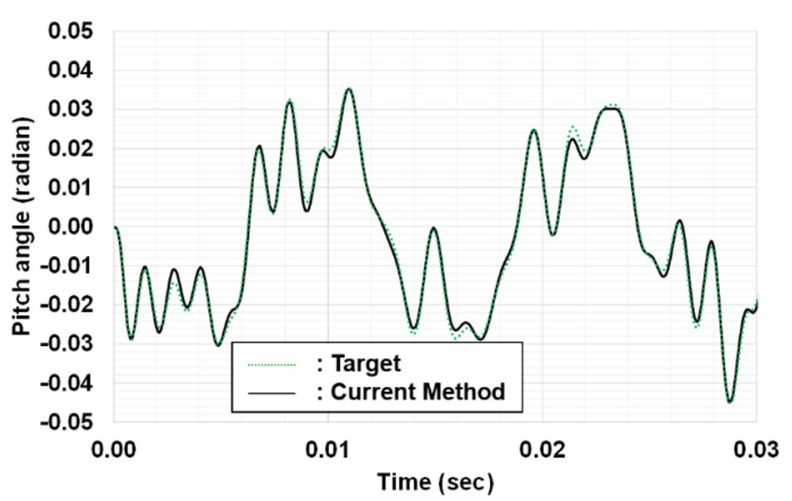

b) Zoom in from 0 to $0.03 \mathrm{~s}$.

Figure 11. Case 2, pitch angle time history at grid 2601.

12

American Institute of Aeronautics and Astronautics 


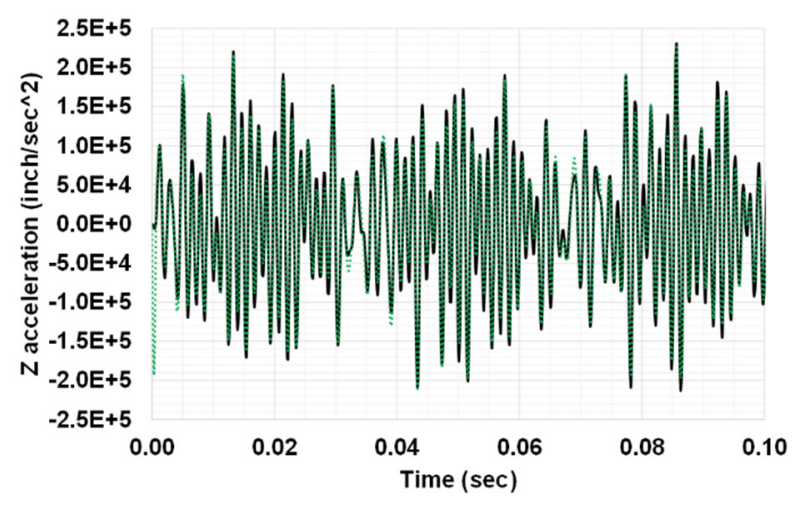

a) From 0 to $0.10 \mathrm{~s}$.

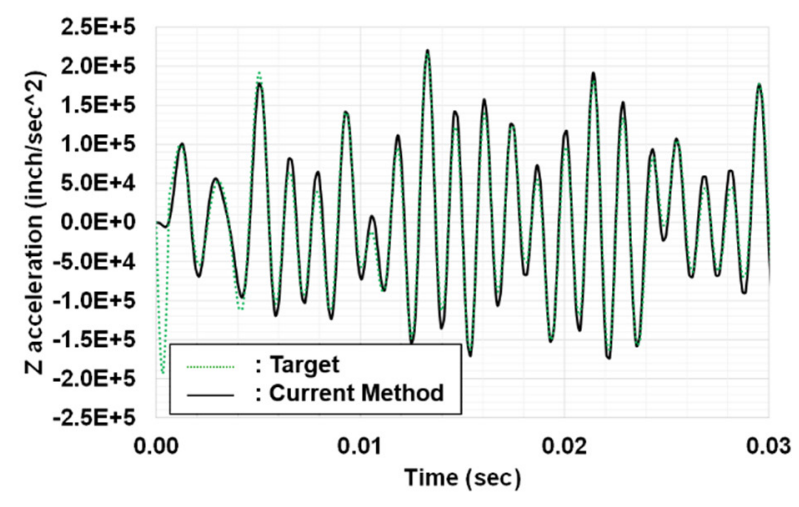

b) Zoom in from 0 to $0.03 \mathrm{~s}$.

Figure 12. Case 2, $\mathrm{Z}$ acceleration time history at grid 2601 .

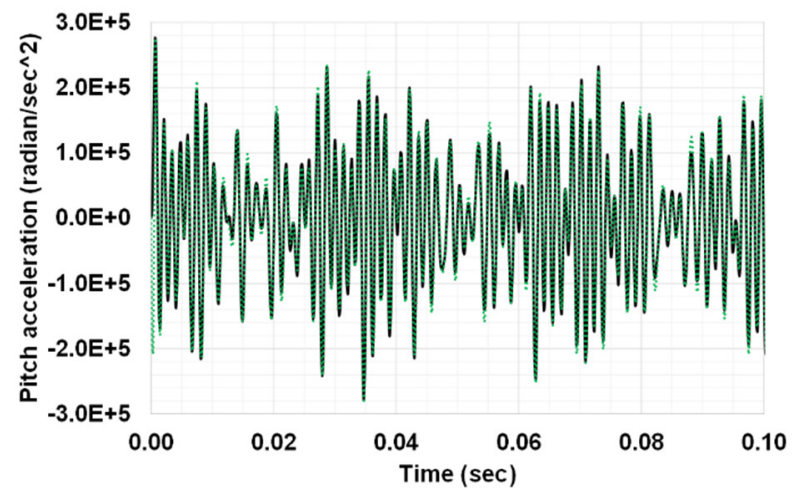

a) From 0 to $0.10 \mathrm{~s}$.

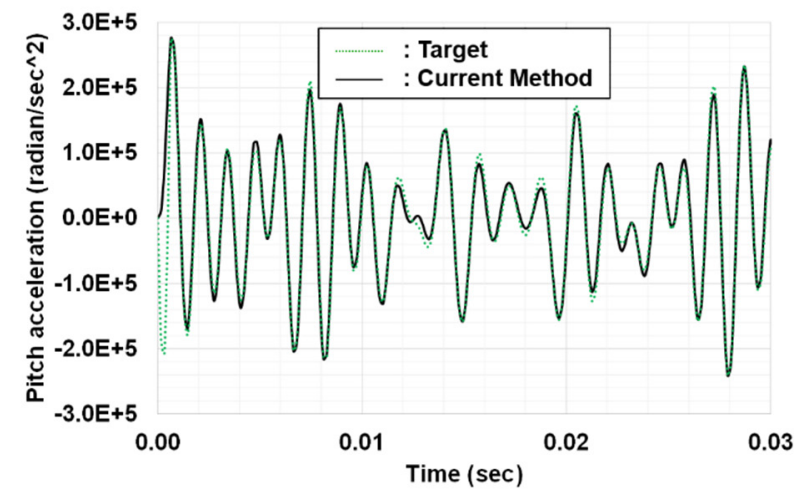

b) Zoom in from 0 to $0.03 \mathrm{~s}$.

Figure 13. Case 2, pitch acceleration time history at grid 2601.

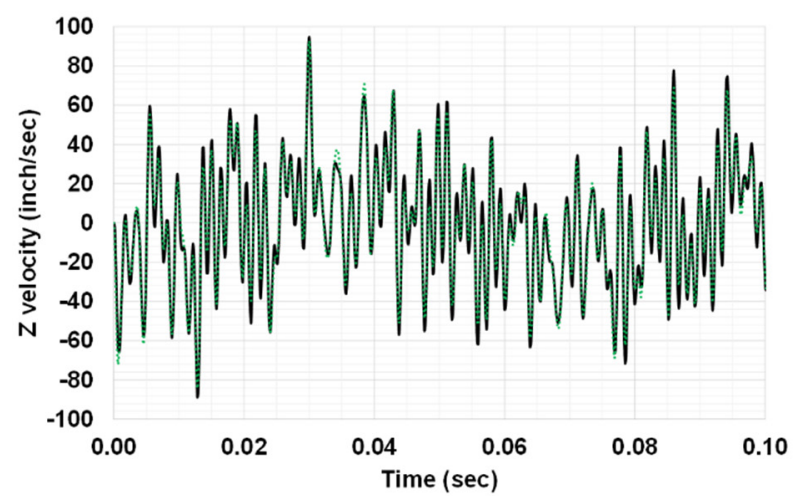

a) From 0 to $0.10 \mathrm{~s}$.

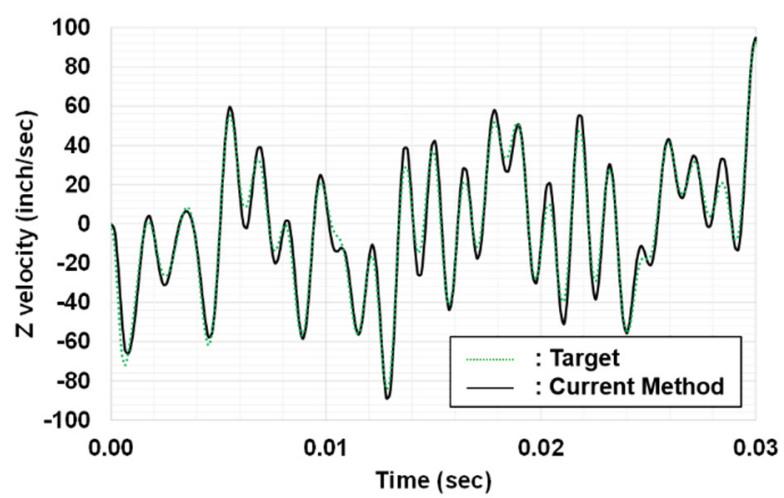

b) Zoom in from 0 to $0.03 \mathrm{~s}$.

Figure 14. Case 2, Z velocity time history at grid 2601. 


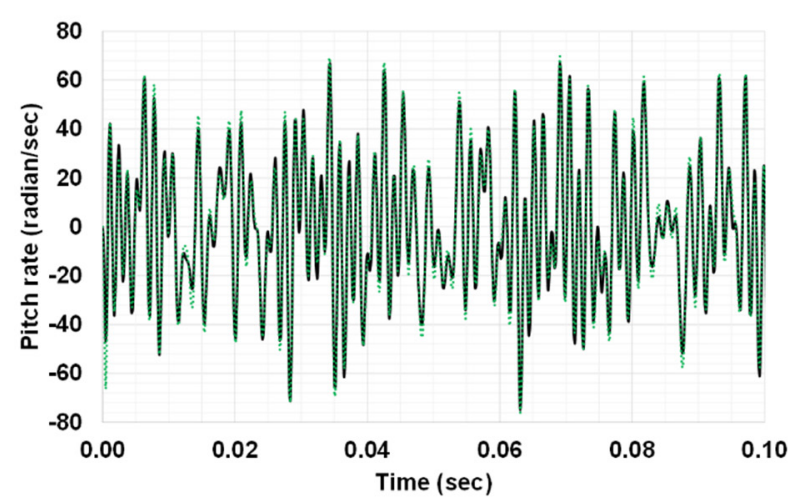

a) From 0 to $0.10 \mathrm{~s}$.

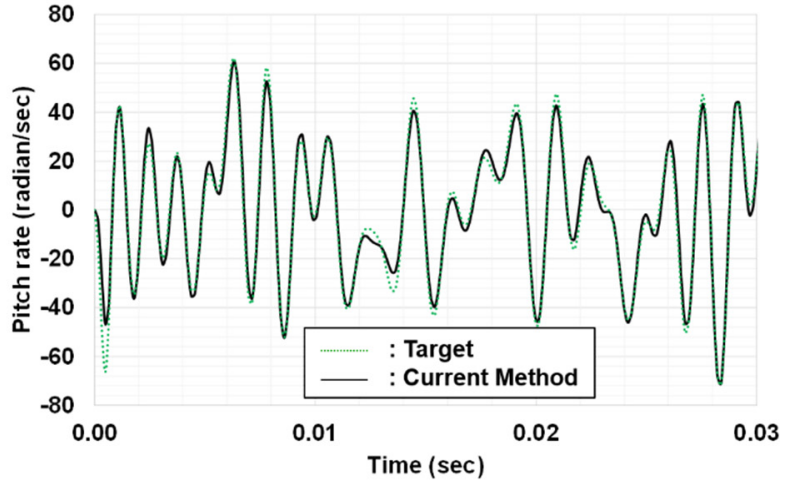

b) Zoom in from 0 to $0.03 \mathrm{~s}$.

Figure 15. Case 2, pitch rate time history at grid 2601.

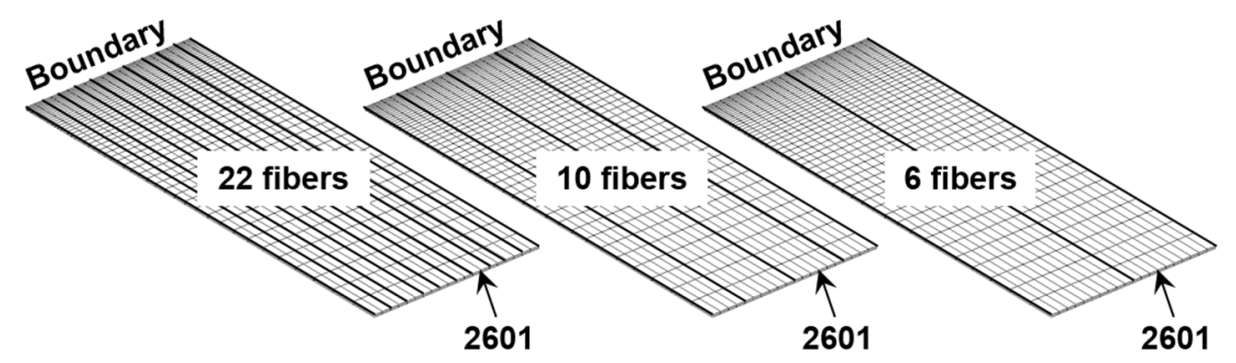

Figure 16. Sensitivity study configurations for number of fibers.

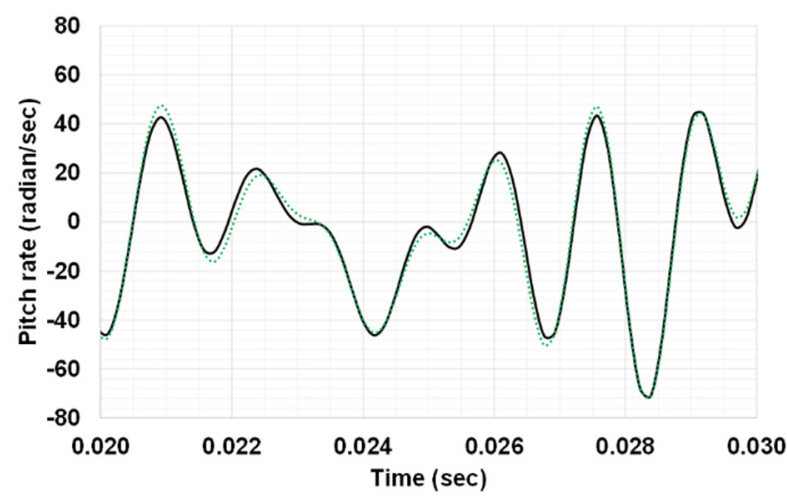

a) Central difference with linear AR model.

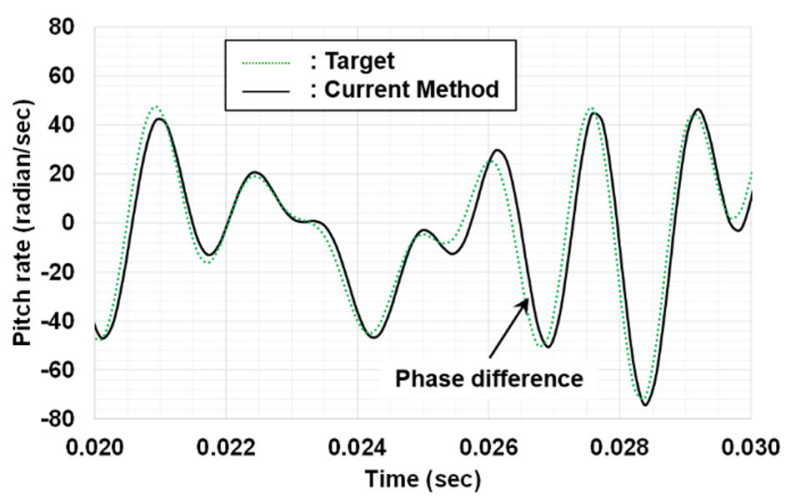

b) Backward difference.

Figure 17. Case 2, pitch rate time history at grid 2601. 


\section{References}

${ }^{1}$ Igawa, H., Murayama, H., Nakamura, T., Yamaguchi, I., Kageyama, K. Uzawa, K., Wada, D., Ohsawa, I., Kanai, M., and Omichi, K., "Measurement of Distributed Strain and Load Identification Using 1500 mm Gauge Length FBG and Optical Frequency Domain Reflectometry," Proceedings of $20^{\text {th }}$ International Conference on Optical Fibre Sensors, Vol. 7503, 2009.

${ }^{2}$ Cusano, A., Cutolo, A., Nasser, J., Giordano, M., and Calabrò A., "Dynamic Strain Measurements by Fibre Bragg Grating Sensor," Sensors and Actuators A: Physical, Vol. 110, No.1-3, 1 February 2004, pp. $276-281$.

${ }^{3}$ Ko, W. L., Richards, W. L., and Tran, V. T., "Displacement Theories for In-Flight Deformed Shape Predictions of Aerospace Structures," NASA TP-2007-214612, 2007.

${ }^{4}$ Kang, L.-H., Kim, D.-K, and Han, J.-J, "Estimation of Dynamic Structural Displacements Using Fiber Bragg Grating Strain Sensors,” Journal of Sound and Vibration, Vol. 305, No. 3, 2007, pp. 534-542.

${ }^{5}$ Moore, J. P., "Method and Apparatus for Shape and End Position Determination Using an Optical Fiber," U.S. Patent No. 7,813,599, issued October 12, 2010.

${ }^{6}$ Shkarayev, S., Krashantisa, R., and Tessler, A., "An Inverse Interpolation Method Utilizing In-Flight Strain Measurements for Determining Loads and Structural Response of Aerospace Vehicles," Proceedings of Third International Workshop on Structural Health Monitoring, 2001.

${ }^{7}$ Tessler, A., and Spangler, J., "A Variational Principle for Reconstruction of Elastic Deformations in Shear Deformable Plates and Shells," NASA/TM-2003-212445, 2003.

${ }^{8}$ Tessler, A., Spangler, J., Mattone, M., Gherlone, M., and Di Sciuva, M., "Real-Time Characterization of Aerospace Structures Using Onboard Strain Measurement Technologies and Inverse Finite Element Method," Proceedings of the 8th International Workshop on Structural Health Monitoring, Vol. 1, 2011, pp. 981-989.

${ }^{9}$ Pak, C.-g., "Wing Shape Sensing from Measured Strain,” AIAA-2015-1427, 2015.

${ }^{10} \mathrm{O}$ 'Callahan, J., Avitabile, P., and Riemer, R., "System Equivalent Reduction Expansion Process," Proceedings of the $7^{\text {th }}$ International Modal Analysis Conference, Las Vegas, Nevada, 1989, pp. 29-37.

${ }^{11}$ Pak, C.-g., and Friedmann, P. P., "New Time Domain Technique for Flutter Boundary Identification," AIAA-92-2102, 1992.

${ }^{12}$ Doggett, R. V. Jr., Rainey, A. G., and Morgan, H. G., “An Experimental Investigation of Aerodynamic Effects of Airfoil Thickness on Transonic Flutter Characteristics," NASA-TMX-79, 1959.

${ }^{13}$ MSC/NASTRAN Quick Reference Guide Version 69, The MacNeal Schwendler Corporation, Newport Beach, California, 1996.

${ }^{14}$ Pak, C.-g., and Truong, S., "Creating a Test-Validated Finite-Element Model of the X-56A Aircraft Structure," Journal of Aircraft, Vol. 52, No. 5, 2015, pp. 1644-1667.

${ }^{15}$ NASA Technical Standard, "Load Analysis of Spacecraft and Payloads," NASA-STD-5002, June 21, 1996. 